\title{
DEFORMATIONS OF COMPLETE MINIMAL SURFACES
}

\author{
HAROLD ROSENBERG
}

\begin{abstract}
A notion of deformation is defined and studied for complete minimal surfaces in $R^{3}$ and $R^{3} / G, G$ a group of translations. The catenoid, Enneper's surface, and the surface of Meeks-Jorge, modelled on a 3-punctured sphere, are shown to be isolated. Minimal surfaces of total curvature $4 \pi$ in $R^{3} / Z$ and $R^{3} / Z^{2}$ are studied. It is proved that the helicoid and Scherk's surface are isolated under periodic perturbations.
\end{abstract}

Let $M$ be a submanifold of a Riemannian manifold $N$ and let $T_{\varepsilon}(M)$ be a tubular neighborhood of $M$ in $N$ of radius $\varepsilon$. A $C^{1}-\varepsilon$ variation of $M$ in $N$ is a submanifold $M_{1} \subset T_{\varepsilon}(M)$ which is a graph over $M$ and is $\varepsilon$ - $C^{1}$-close to $M$. This means $M_{1}$ is pointwise $\varepsilon$-close to $M$ in each fibre of $T_{\varepsilon}(M)$ and the tangent planes as well. We are interested in complete minimal submanifolds $M$ of $N$ (abbreviated c.m.s.) and $C^{1}-\varepsilon$ variations which are also c.m.s.'s, and henceforth we always assume $M$ and $M_{1}$ are c.m.s.'s. We say $M$ is isolated if for some $\varepsilon>0$, the only $\varepsilon$ - $C^{1}$-variations of $M$ differ from $M$ by an ambient isometry of $N$. In this paper we shall study this question when $N$ is $R^{3}$ or a translation space: $R^{3}$ modulo a group of translations.

A flat plane in $R^{3}$ is isolated. This follows immediately from Bernstein's theorem: a function of two variables on $R^{2}$ whose graph is a c.m.s. is linear. We shall prove Enneper's surface, the catenoid, and a certain 3-punctured sphere, discovered by Meeks and Jorge, are isolated in $R^{3}$. We prove the helicoid and Scherk's surface are isolated under periodic deformations, i.e., the helicoid of total curvature $4 \pi$ in $R^{3}$ modulo one translation is isolated in this translation space and Scherk's surface of total curvature $4 \pi$ is isolated in $R^{3}$ modulo two translations. This Scherk surface is conformally a 4-punctured sphere and the helicoid a 2-punctured sphere.

We shall study minimal submanifolds of translation spaces. Many of the techniques developed by $\mathbf{R}$. Osserman can be adapted to this context and yield information about periodic minimal surfaces in $R^{3}$. For example, we classify c.m.s.'s of total curvature $4 \pi$ in translation space. In $R^{3}$, Osserman has proved the catenoid and Enneper's surface are the only c.m.s.'s of total curvature $4 \pi$. We obtain an analogous classification in $R^{3} / G$. In §VI, we describe these surfaces for $G=Z$.

We study c.m.s.'s $M$ of finite total curvature in translation spaces. We prove a deformation (i.e., an $\varepsilon$ - $C^{1}$-variation) $M_{1}$ of a finite total curvature c.m.s. in a translation space is also of finite total curvature, and is conformally a compact

Received by the editors December 2, 1983 and, in revised form, May 1, 1984.

1980 Mathematics Subject Classification (1985 Revision). Primary 53A10. 
Riemann surface $\bar{M}_{1}$ punctured in a finite number of points. The Gauss map $g_{1}$ of $M_{1}$ and the analytic differential $\omega_{1}$ of the Weierstrass representation of $M_{1}$, extend to a meromorphic function $\bar{g}_{1}$ on $\bar{M}_{1}$ and a meromorphic differential $\bar{\omega}_{1}$ on $\bar{M}_{1}$. The degree of $\bar{g}_{1}$ is the same as the degree of $\bar{g}$, the extended Gauss map of the unperturbed surface $M$. This reduces the study of deformations of finite total curvature $M$ to the study of pairs $(\bar{g}, \bar{\omega})$ on $\bar{M}_{1}$ with relations between the poles of $\bar{g}$ and the zeros of $\bar{\omega}$ and other relations coming from closeness in space and residue conditions. In general, this study seems difficult.

The Gauss map $g$ of a nonflat c.m.s. of finite total curvature in $R^{3}$ can miss at most 3 points of the Riemann sphere $S$ [4]. It is not known if 3 is sharp. Osserman's proof shows that $g$ can miss at most 4 points for a nonflat c.m.s. of finite total curvature in a translation space. Four is sharp as Scherk's surface shows. This has already been observed by Gacksatter [1]; he calls such surfaces abelian (Lawson calls them algebraic [2]). We show the Gauss maps of deformations of these surfaces have the same image. Xavier has proved the Gauss map of a c.m.s. in $R^{3}$ can miss at most 7 points (unless it is flat) [6]. It is not known if 7 can be reduced to 4 .

We would very much like to know if the helicoid is isolated in $R^{3}$. We prove in $\S$ VII that certain deformations of the helicoid do not exist. It seems plausible that any c.m.s. conformally parametrised by $\mathbf{C}$ is isolated.

We originally proved the catenoid is isolated in collaboration with Remi Langevin. Discussions with Mike Beeson, Bill Meeks, Rich Schoen and Dennis Sullivan have been very helpful in the preparation of this paper.

Some remarks are in order concerning the definition of an isolated surface. When trying to understand something, it is natural to study a neighborhood of the object in question. Complete minimal surfaces are particularly mysterious (alas, their deformations as well). Weierstrass introduced a deformation of minimal surfaces (known to specialists as the Weierstrass deformation) and this has certainly proved useful. The one parameter family of (locally) isometric surfaces joining the helicoid to the catenoid can be realised as a Weiertrass deformation. However, the convergence here is analogous to the manner by which a family of circles can be made to converge to a line; certainly no circle is in a tubular neighborhood of the line of fixed radius. It seems natural to consider neighborhoods defined by tubular neighborhoods of a fixed radius. $C^{i}$-close is perhaps superfluous but our techniques require this for the time being.

When this paper was written, we had no example of a c.m.s. in $R^{3}$ which was not isolated. Subsequently, the author, in collaboration with E. Toubiana, found such an example. In principal, our paper describing this example is in the same issue of the Transactions as the present paper. Robert Bryant has brought to our attention that this deformable example occurs in his paper on higher critical values of the Willmore functional (preprint). He was not studying deformations but his examples are very interesting in the context of deformations.

I. The Weierstrass representation in translation spaces. Let $M$ be a minimal surface in $R^{3}$. M admits local parametrisations $X: D \rightarrow M, D$ an open subset of $C$ and $X$ a conformal map with harmonic coordinate functions; such parametrisations 
are equivalent to minimality. Write $z=u+i v, X(z)=\left(x_{1}(z), x_{2}(z), x_{3}(z)\right) . X$ conformal means $X_{u} \perp X_{v}\left(X_{u}\right.$ means partial derivative with respect to $\left.u\right)$ and $\left\|X_{u}\right\|=\left\|X_{v}\right\|$. Harmonicity means $\left(\partial^{2} x_{k}\right) /\left(\partial u^{2}\right)+\left(\partial^{2} x_{k}\right) /\left(\partial v^{2}\right)=0, k=1,2,3$. Let $\phi_{k}(z)=\left(\partial x_{k}\right) /(\partial u)-i\left(\partial x_{k}\right) /(\partial v)$, so that $\phi_{k}$ are analytic in $D$, (i) $\phi_{1}^{2}+\phi_{2}^{2}+$ $\phi_{3}^{2} \equiv 0$ and (ii) $\left|\phi_{1}\right|^{2}+\left|\phi_{2}\right|^{2}+\left|\phi_{3}\right|^{2} \neq 0$. Clearly if we are given analytic functions $\phi_{k}$ in $D$, satisfying (i) and (ii), then we obtain a parametrised (by $D$ ) minimal surface by defining $x_{k}(z)=\operatorname{Re} \int_{z_{0}}^{z} \phi_{k}(z) d z, z_{0} \in D, k=1,2,3$. The forms $\phi_{k}(z) d z$ do not depend on the local parameter, i.e., if $\tilde{X}: \tilde{D} \rightarrow M$ is a conformal map with harmonic coordinate functions, then $\phi_{k}(z) d z=\tilde{\phi}_{k}(\tilde{z}) d \tilde{z}, z \rightarrow \tilde{z}$ the change of coordinates. So a minimal surface determines three global analytic forms $\phi_{k}(z) d z$ satisfying (i) and (ii). Moreover the forms have no real periods on $M$ since the coordinate functions are single valued on $M$. Conversely, if we are given three forms on $M$ satisfying (i), (ii) and the period relations, then we obtain a conformal minimal immersion $M \stackrel{X}{\rightarrow} R^{3}$ by integration, i.e., $x_{k}=\operatorname{Re} \int \phi_{k}(z) d z$. Changing the base point in the integral changes the immersion by a translation. Now (assuming $M$ is not a flat plane) the data: three analytic forms on $M$ satisfying (i) and (ii) are equivalent to a pair $(g, \omega)$, where $g$ is a meromorphic function on $M$ to $S, \omega$ is an analytic differential (i.e. an analytic one-form) satisfying: the poles of $g$ are precisely the zeros of $\omega$ and the order of a pole of $g$ is one half the order of this zero of $\omega$. The relation is

$$
\begin{array}{llrl}
\phi_{1} & =\frac{1}{2}\left(1-g^{2}\right) \omega, & & \omega=\phi_{1}-i \phi_{2}, \\
\phi_{2} & =\frac{i}{2}\left(1+g^{2}\right) \omega, & & \text { or } \\
\phi_{3} & =g \omega . & &
\end{array}
$$

Thus a minimal surface $M$ in $R^{3}$ is determined by such pairs $(g, \omega)$, provided the residue conditions are satisfied for the $\phi_{k}$, i.e. they have no real periods on $M$. This is the usual Weierstrass representation of minimal surfaces in $R^{3}$. We mention that the metric on $M$ is given by $d s=\lambda|d z|$, where $\lambda=\left(1+|g|^{2}\right)|\omega| / 2$, and $g$ is the Gauss map.

Now let $G$ be a group of translations of $R^{3}$ and let $N=R^{3} / G, p: R^{3} \rightarrow N$ the projection. Let $M$ be a minimal submanifold of $N$ and $M_{1}$ be a connected component of $p^{-1}(M)$. Let $\left(g_{1}, \omega_{1}\right)$ be the pair associated to $M_{1}$ via the Weierstrass representation. Since parallel translation in $R^{3}$ leaves invariant the normals, and $g_{1}$ is the Gauss map of $M_{1}, g$ passes to a meromorphic map $g: M \rightarrow S$. Notice that the forms $\phi_{k}^{1}$ on $M_{1}, k=1,2,3$, also pass to the quotient. To see this, let $p \in M_{1}$, $\gamma \in G$ and $\xi: D \rightarrow M_{1}$ be a local parameter at $p$. So

$$
\phi_{k}^{1}(z) d z=\left(\frac{\partial x_{k}^{1}}{\partial u}-i \frac{\partial x_{k}^{1}}{\partial v}\right) d z
$$

in $D$. This does not depend on the local parameter at $p$, so to check that $\phi_{k}^{1}(p)=\phi_{k}^{1}(\gamma(p))$ we can choose any local parameter at $\gamma(p)$. We use $\gamma \cdot \xi: D \rightarrow M_{1}$ 
as a local parameter at $\gamma(p)$. Then

$$
\phi_{k}^{1}(\gamma(p))=\left(\frac{\partial x_{k}^{1}(\gamma(p))}{\partial u}-i \frac{\partial x_{k}^{1}(\gamma(p))}{\partial v}\right) d z
$$

Since $\gamma$ is a translation, the partial derivatives are the same at $p$ and $\gamma(p)$. Thus $\omega_{1}$ passes to an analytic differential $\omega$ on $M$. Thus $M$ also has a Weierstrass representation $(g, \omega)$ and the poles of $g$ correspond to the zeros of $\omega$ with the proper multiplicities as before. However, the coordinate functions $x_{k}=\operatorname{Re} \int \phi_{k}$ need not be single valued on $M$. Many examples will be given in $\S \mathrm{VI}$; periodic minimal surfaces in $R^{3}$ are the most interesting. Any minimal surface in $R^{3}$ yields a minimal surface in $R^{3} / G$ by projection, however periodic minimal surfaces may be simpler in the quotient. Infinite total curvature may become finite, e.g. the helicoid in $R^{3} / Z$ and Scherk's surface in $R^{3} / Z^{2}$.

II. Finite total curvature. Let $G$ be a group of translations of $R^{3}$ and let $M \subset R^{3} / G$ be a c.m.s. of finite total curvature. Then there is a compact Riemann surface $\bar{M}$ and $M$ is conformally equivalent to $\bar{M}$ punctured at a finite number of points $p_{1}, \ldots, p_{r}$ [2]. We observe that (as in $\left.R^{3}\right)$ the pair $(g, \omega)$ extends to a meromorphic map $g: \bar{M} \rightarrow S^{2}$ and a meromorphic differential $\omega$ on $\bar{M}$. To see that $g$ extends to $\bar{M}$, notice that the total curvature of $M$ is the area of its spherical image under $g$, counted with multiplicity; hence each value is assumed at most a finite number of times; cf. [1]. Now $M$ is finitely connected and each end of $M$ is conformally a once punctured disc. The puncture cannot be an essential singularity of $g$ since then $g$ would take on values infinitely often in a neighborhood of the puncture. Therefore $g$ extends to a meromorphic map at the puncture, thus to $\bar{M}$. Now the analytic differential $\omega$ also extends to a meromorphic differential on $\bar{M}$, having a pole at least of order one at each puncture (Scherk's surface in $R^{3} / Z^{2}$ shows that the poles need not be of order 2 at the punctures as in $R^{3}$ ).

Let $D$ be a punctured disc, representing an end of $M, D=\{z \in \mathrm{C} / 0<|z|<1\}$ and the puncture is the origin. Write $\omega=f(z) d z, f$ analytic in $D$. Then the metric on $M$ in $D$ takes the form $d s=\lambda|d z|$, where $\lambda=|f|\left(1+|g|^{2}\right) / 2$. We can assume the origin is not a pole or zero of $g$ (by eventually making a preliminary rotation of $M$ and replacing $G$ by the conjugate group). Then completeness of $M$ implies $\int_{\gamma}|f(z)||d z|=\infty$ for every path $\gamma$ tending to the origin. Then $f$ has a pole at 0 , at least of order one [4]. Thus $\omega$ also extends to $\bar{M}$.

III. Deformations of finite total curvature surfaces. Let $M_{0}$ be a c.m.s. in $R^{3} / G$ and let $M$ be an $\varepsilon$ deformation of $M_{0}$. Orthogonal projection of $M$ to $M_{0}$ is a quasiconformal homeomorphism. Thus if $M_{0}$ is conformally parametrised by $\mathrm{C}$ then so is $M$, e.g. the helicoid, catenoid and Enneper's surface. This idea applies to finite total curvature minimal surfaces and we discuss this now.

Suppose $M_{0}$ is of finite total curvature, so that $M_{0}$ is conformally equivalent to a compact Riemann surface $\bar{M}_{0}$ punctured at a finite number of points $p_{1}, \ldots, p_{r}$. We claim that the same holds for deformations $M$ of $M_{0}$. First we shall prove that $M$ is also conformally a compact Riemann surface $\bar{M}$ punctured at $r$ points $(\bar{M}$ is 
homeomorphic to $\bar{M}_{0}$ ). Let $E_{0}$ be an end of $M_{0} ; E_{0}$ is conformally a once punctured disc and $g_{0}$ extends across the puncture. Let $E$ be the corresponding end of $M$; we need to prove $E$ is also conformally a once punctured disc. After a preliminary rotation of $M_{0}$ (hence of $M$ and $G$ ) we may assume $g_{0}$ has no poles in $E_{0}$, nor $g$ in $E$. An open subset $A$ of $\mathbf{C}$ is called hyperbolic if $A$ admits Green's functions, or equivalently, if $\log \left(1+|z|^{2}\right)$ has a harmonic majorant in $A$ [4]; otherwise $A$ is called parabolic. Clearly an open subset of a hyperbolic set is hyperbolic. Now $g_{0}$ and $g$ are open maps, hence $g(E)$ is hyperbolic since $g_{0}(E)$ is contained in the complement of a closed disc (which is hyperbolic) and $g(E)$ is close to $g_{0}(E)$, hence $g(E)$ is an open subset of a hyperbolic set. Therefore it suffices to prove:

Lemma. An end of $M$, whose spherical image is hyperbolic, is conformally a once punctured disc.

Proof. Let $E=\{z \in \mathbf{C} / 0 \leqslant r<|z|<1\}$ and $d s=\lambda|d z|$ be the induced metric in $E$. We know, by completeness of $M$, that for all paths $z(t)$ in $E$ such that $\lim _{t \rightarrow 1}|z(t)|=r$, we have $\int_{z(t)} d s=\infty$. Thus by [4], we have $r=0$ provided we can find a harmonic $h(z)$ such that $\log \lambda(z) \leqslant h(z)$ in $E$. Here is how we find $h(z)$. We know $\lambda=|f|\left(1+|g|^{2}\right) / 2$, and since $g(E)$ is hyperbolic, there is a harmonic $h_{0}$ on $g(E)$ such that

$$
\log \left(1+|g(z)|^{2}\right) \leqslant h_{0}(g(z)) \text { for } z \in E .
$$

Now $\lambda \leqslant \tilde{\lambda}=\frac{1}{2}|f| e^{h_{0}(g(z))}$ so

$$
\log \lambda \leqslant \log |f|^{2}+h_{0}(g(z)) .
$$

Since $g$ has no poles in $E$, we have $f(z) \neq 0$ in $E$. Thus $h(z)=\log |f(z)|^{2}+$ $h_{0}(g(z))$ is a harmonic majorant of $\log \lambda$ in $E$ and $r=0$.

Now to prove $M$ has finite total curvature it suffices to prove $g$ extends to $\bar{M}$ [2]. Let $E$ be a punctured disc end of $M$. If the puncture were an essential singularity of $g$, then $g$ would take on almost every value in a neighborhood of the puncture. However $g(E)$ is contained in a small disc of $S$ since it is close to the image of the unperturbed end. Thus the puncture is a removable singularity.

Next we shall prove the degree of $g: \bar{M} \rightarrow S$ is the same as the degree of $g_{0}$ : $\bar{M}_{0} \rightarrow S$. Suppose the degree of $g_{0}$ is $l$, so that the preimage of a point of $S$ is $l$ points of $\bar{M}_{0}$, counted with multiplicity. Let $z \in S$ be a regular point of $g_{0}$, so that $g_{0}^{-1}(z)$ consists of $l$ points $k_{1}, \ldots, k_{l}$ and each $k_{j}$ has a disc neighborhood $D_{j}$ mapped homeomorphically onto a disc neighborhood $D$ of $z$. We choose $z$ so that no $k_{j}$ is a puncture of $M_{0}$. Let $\tilde{D}_{j}$ be the disc on $M$ obtained by orthogonal projection of $D_{j}$ onto $M$. For $\varepsilon$ sufficiently small, $g\left(\partial \tilde{D}_{j}\right)$ can be made close enough to $g_{0}\left(\partial \tilde{D}_{j}\right)$ so that $g \tilde{D}_{j}$ contains $z$. Hence each $\tilde{D}_{j}$ contains one preimage of $z$ and $\operatorname{deg} g \geqslant \operatorname{deg} g_{0}$. Now consider $F=\bar{M}_{0}-\bigcup_{j=1}^{l}$ int $D_{j}$. Since $g_{0} F$ is far from $z$, we can make $g\left(\bar{M}-\bigcup_{j=1}^{l}\right.$ int $\left.\tilde{D}_{j}\right)$ disjoint from $z$ for small perturbations. Hence $\operatorname{deg} g=\operatorname{deg} g_{0}$. 
IV. Some isolated minimal surfaces. Robert Osserman has proved that Enneper's surface and the catenoid are the only c.m.s.'s in $R$ of total curvature $4 \pi$ [4]. Now we know that a deformation of a finite total curvature c.m.s. has the same total curvature since the Gauss maps have the same degree. Therefore Enneper's surface and the catenoid are isolated. For completeness we prove directly that these surfaces are isolated.

The Weierstrass representation of Enneper's surface $M_{0}$ is $\left(g_{0}, \omega_{0}\right)$, where $M_{0}$ is parametrised by $\mathbf{C}, g_{0}(z)=z, \omega_{0}=d z$. Now if $M$ is a deformation of $M_{0}$, the conformal type of $M$ is also $\mathbf{C}$ and $M$ is given by a conformal immersion $X: \mathbf{C} \rightarrow M$ with harmonic coordinate functions. The limiting value of the normal to $M$ at its unique end is the same as that of $M_{0}$, i.e. $(0,0,1)$, so $g(\infty)=\infty$ and after reparametrising $M$ by composing $X$ with a Moebius transformation $S \rightarrow S$, leaving $\infty$ fixed, we may suppose $g(z)=z$ (since $g: S \rightarrow S$ is a bijective meromorphic map leaving $\infty$ fixed, so of the form $a z+b$ ). Now $\omega$ is an analytic differential on $\mathbf{C}$ with no zeros or poles and $\omega$ extends to a meromorphic differential on $S$. Therefore $\omega=c d z$ for some $c \in \mathbf{C}$. It is a remarkable fact that for $c=e^{i \theta}$ the surfaces $\{g(z)=z, \omega=d z\}$ and $\{g(z)=z, \omega=c d z\}$ are congruent in $R^{3}$; they differ by rotation by $\theta / 2$. So for general $c \in \mathbf{C}, g(z)=z, \omega=c d z$ differs from $\{g(z)=z$, $\omega=d z\}$ by a rotation followed by a homothety. Hence for $M$ we must have $c=1$ and $M=M_{0}$.

Next consider the catenoid $M_{0} . M_{0}$ can be described by a conformal embedding $X_{0}: S-\{0, \infty\} \rightarrow R^{3}$ with $g_{0}(z)=z, \omega_{0}=d z / z^{2}$. Let $M$ be a deformation of $M_{0}$. $M$ is also conformally a 2-punctured sphere so we have a conformal embedding $X$ : C $-0 \rightarrow M$ with harmonic coordinate functions. The values of the Gauss map $g$ are the same as $g_{0}$ at the punctures $0, \infty$, thus, after reparametrising, $g$ may be taken to be $g(z)=z$. Now $\omega$ is a meromorphic differential on $S$ with no zeros in $\mathbf{C}$, and analytic in $\mathbf{C}$, hence $\omega=c d z / z^{k}$ for some $c \in \mathbf{C}$ and integer $k$. We have $d s=\lambda|d z|$ $=|\omega|\left(1+|g|^{2}\right) / 2$. By completeness of $M$ at 0 we have $k>0$ and by completeness at $\infty$ we have $k<4$. A residue calculation shows $k \neq 1$. Indeed, if $k=1$, then $\operatorname{Res}(\phi, 0)=c / 2$ and $\operatorname{Res}\left(\phi_{2}, 0\right)=i c / 2$. Since $x_{1}$ and $x_{2}$ are single valued on $M$ we conclude $c$ is both real and pure imaginary, hence $c=0$, a contradiction. A similar residue calculation shows $k \neq 3$. So $\omega=c d z / z^{2}$. Now $x_{3}=\operatorname{Re} \int^{z} c d z / z$ is single valued so $\operatorname{Res}\left(\phi_{3}, 0\right)=c$ must be real. Thus $M$ differs from $M_{0}$ by a dilation and since they are close in space we have $c=1$. Therefore $M$ is a catenoid.

Meeks and Jorge have discovered interesting examples of finite total curvature c.m.s.'s which are modelled on an $n$-punctured sphere [3] for any integer $n$. The Weierstrass representation of this surface is $g(z)=z^{n-1}, \omega=d z /\left(z^{n}-1\right)^{2}$, where $M=S$ punctured at the $n$ roots of unity. For $n=2$, this gives a catenoid (rotated from our previous discussion). In general, this surface is immersed and has $n$ embedded "catenoid type" ends.

THEOREM. The c.m.s. $M_{0}: g_{0}(z)=z^{2}, \omega_{0}=d z /\left(z^{3}-1\right)^{2}$, is isolated.

Proof. Let $M$ be a deformation of $M_{0} . M$ has total curvature $8 \pi$; the Gauss map $\bar{M} \rightarrow S$ is a rational map of degree 2. So $g(z)=R(z) / S(z), R$ and $S$ polynomials 
and $\max \operatorname{deg}\{R, S\}=2$. Since three punctured spheres are all conformally equivalent, we can parametrise $M$ by $S-\left\{1, \alpha_{1}, \alpha_{2}\right\}, \alpha_{k}$ the cube roots of unity. We know $g$ takes the same values as $g_{0}$ at the punctures, so $g(1)=1, g\left(\alpha_{1}\right)=\alpha_{2}, g\left(\alpha_{2}\right)=\alpha_{1}$. There are five cases to consider: $1 . g$ is a polynomial of degree $2 ; 2 . R$ is constant and $\operatorname{deg} S=2 ; 3 . \operatorname{deg} R=2$ and $\operatorname{deg} S=1 ; 4 . \operatorname{deg} R=1$ and $\operatorname{deg} S=2$; and 5 . $\operatorname{deg} R=\operatorname{deg} S=2$; this is the difficult one. Let $\omega=P(z) d z / Q(z), P, Q$ polynomials.

1. Clearly $g(z)=z^{2}$ since $g$ takes the prescribed values at $1, \alpha_{1}, \alpha_{2}$. We know $\omega$ has zeros (in $M$ ) only at the poles of $g$ so $P(z)$ is constant. Moreover $\infty$ is a regular point of $M$ so a zero of $\omega$ of order 4. It follows that $\operatorname{deg} Q(z)=6$. In $R^{3}$, it is a general fact that $\omega$ has a pole at each puncture (where $g$ takes a finite value) at least of order 2 [4]. Therefore

$$
\omega=\frac{c d z}{(z-1)^{2}\left(z-\alpha_{1}\right)^{2}\left(z-\alpha_{2}\right)^{2}}
$$

for some $c \in \mathbf{C}$. Now $x_{3}$ is a single valued function on $M$ and a calculation of $\operatorname{Res}\left(\phi_{3}, 1\right)$ shows $c$ is real. Therefore $M$ differs from $M_{0}$ by a dilation and since they are $\varepsilon$ close we have $c=1, M=M_{0}$.

We leave case 2 to the reader.

3. A calculation yields $g(z)=\left(z^{2}-\alpha^{2}\right) /\left(-\alpha^{2} z+1\right)$ for some $\alpha \in \mathbf{C}, \alpha \neq \pm 1$. Hence the zeros of $\omega$ are precisely the points $\beta=1 / \alpha^{2}$ and $\infty$ and each is of order 2 since they are simple poles of $g$. Thus $P(z)=c(z-\beta)^{2}$ and degree $Q(z)=6$. Since $\omega$ has poles at least of order 2 at each puncture, we have

$$
\omega=c(z-\beta)^{2} /\left(z^{3}-1\right)^{2} .
$$

We will prove this is impossible by doing the residue calculations at $z=1$. Since $x_{1}$, $x_{2}$ are single valued functions on $M$ we have $\int_{\gamma} \omega=\overline{\int_{\gamma} \omega g^{2}}$, where $\gamma=\{z /|z-1|=$ $.2\}$. These equations yield:

$$
c \beta(1-\beta)=\bar{c}(1-\bar{\beta})(1+\bar{\beta}) .
$$

Now $x_{3}$ single valued on $M$ means $g \omega$ has real residues. A calculation at $z=1$ yields $c(1-\beta)$ is real. Combining this with (I) we conclude $\beta=1+\bar{\beta}$ or $c(1-\beta)$ $=0$. Both are clearly impossible.

4. We have $g(z)=(a z+b) /(z-\alpha)(z-\beta)$. The equations $g(1)=1, g\left(\alpha_{1}\right)=\alpha_{2}$, $g\left(\alpha_{2}\right)=\alpha_{1}$, imply $\alpha=0$ or $\beta=0$, and $a=1-\alpha \beta, b=2 \alpha \beta-\alpha-\beta$. This gives $g(z)=1 / z$ which contradicts $\operatorname{deg} Q=2$.

Finally we must treat case $5, \operatorname{deg} R(z)=\operatorname{deg} S(z)=2$. Let $\alpha, \beta$ be the poles of $g$, i.e. the roots of $Q$. An easy calculation shows

$$
g(z)=\left(\alpha \beta z^{2}+z-(\alpha+\beta)\right) /(z-\alpha)(z-\beta) .
$$

Clearly $\alpha, \beta$ are not cube roots of unity so $\omega$ has double zeros at $\alpha$ and $\beta$ and no others. Also $\omega$ has poles at the punctures at least of order 2 hence $\operatorname{deg} Q(z) \geqslant 6$. 
Since $\infty$ is not a zero of $\omega$, we have $\operatorname{deg} Q(z)=6(\operatorname{deg} P(z)=4)$. Hence

$$
\omega=\frac{c(z-\alpha)^{2}(z-\beta)^{2}}{\left(z^{3}-1\right)^{2}} d z
$$

We now list the residue calculations, and we leave the calculations to the courageous reader. The $x_{1}, x_{2}$ well-defined functions are equivalent to $\int \omega=\overline{\int \omega g^{2}}$ on all loops.

Equations (I) and (II) are this integral equation at $z=1$ and $\alpha_{1}$ respectively:

$$
c(1-\alpha)(1-\beta)(1-\alpha \beta)=-\bar{c}(1-\bar{\alpha})(1-\bar{\beta})(\bar{\alpha}+\bar{\beta}+\bar{\alpha} \bar{\beta}),
$$

$c\left(\alpha_{1}-\alpha\right)\left(\alpha_{1}-\beta\right)\left(\alpha_{2}-\alpha \beta\right) \alpha_{1}=-\bar{c}\left(\bar{\alpha} \bar{\beta} \alpha_{1}+\alpha_{2}-(\bar{\alpha}+\bar{\beta})\right)\left(\bar{\alpha} \bar{\beta} \alpha_{1}+(\bar{\alpha}+\bar{\beta}) \alpha_{2}\right)$.

We use related equations obtained as follows. Expand $g(z)$ and $\omega=f(z) d z$ in power series at $z=1$ :

$$
g(z)=g(1)+g^{\prime}(1) w+o\left(w^{2}\right), \quad f_{1}(z)=f_{1}(1)+f_{1}^{\prime}(1) w+o\left(w^{2}\right),
$$

where

$$
f_{1}(z)=\frac{c(z-\alpha)^{2}(z-\beta)^{2}}{\left(z-\alpha_{1}\right)^{2}\left(z-\alpha_{2}\right)^{2}} \quad \text { and } \quad w=z-1
$$

Then

$$
\begin{aligned}
\phi_{1} & =\frac{f}{2}\left(1-g^{2}\right)=\frac{1}{2}\left(f(1)+\frac{f_{1}^{\prime}(1)}{w}+o(1)\right)\left(-2 g^{\prime}(1) w+o\left(w^{2}\right)\right) \\
& =-\frac{f_{1}(1) g^{\prime}(1)}{w}+o(1)
\end{aligned}
$$

and

$$
x_{1}=\operatorname{Re} \int \phi_{1}=-\operatorname{Re}\left(f_{1}(1) g^{\prime}(1) \log w+o(w)\right),
$$

so $x_{1}$ single valued implies $f_{1}(1) g^{\prime}(1)$ is real, i.e.

$$
c(1-\alpha)(1-\beta)(2 \alpha \beta-1+(\alpha+\beta))
$$

is real. A similar calculation with $\phi_{2}=i f\left(1+g^{2}\right) / 2$ shows $f_{1}(1) g^{\prime}(1)+f_{1}^{\prime}(1)$ is pure imaginary, and this easily implies $c(1-\alpha)(1-\beta)(1+\alpha+\beta)$ is pure imaginary.

The $x_{3}$ residue condition at $z=1$ is $c(1-\alpha)(1-\beta)(1+\alpha+\beta)$ is real. Thus $c(1-\alpha)(1-\beta)(1+\alpha+\beta)=0$, hence $\alpha+\beta=-1$ since neither $\alpha$ or $\beta$ is 1 and $c \neq 0$.

Now consider $\phi_{3}$ near $\alpha_{1}$. We have

$$
\phi_{3}=f g=\left(\frac{h\left(\alpha_{1}\right)}{u^{2}}+\frac{h^{\prime}\left(\alpha_{1}\right)}{u}+o(1)\right)\left(\alpha_{2}+g^{\prime}\left(\alpha_{1}\right) u+o\left(u^{2}\right)\right) \text {, }
$$

where $u=z-\alpha_{1}, \quad h(z)=c(z-\alpha)^{2}(z-\beta)^{2} /(z-1)^{2}\left(z-\alpha_{2}\right)^{2}$. Then since $\operatorname{Res}\left(\phi_{3}, \alpha_{1}\right)$ is real we conclude: $h\left(\alpha_{1}\right) g^{\prime}\left(\alpha_{1}\right)+h^{\prime}\left(\alpha_{1}\right) \alpha_{2}$ is real. This yields $c\left(\alpha_{1}-\alpha\right)\left(\alpha_{1}-\beta\right)$ is pure imaginary. Now substitute this last relation into (II) and use the fact $\alpha \beta \alpha_{2}+\alpha_{1}+1=\left(\alpha_{1}-\alpha\right)\left(\alpha_{1}-\beta\right) \alpha_{2}$ to obtain $2 \alpha_{2}=\alpha \beta+\overline{\alpha \beta}$. This is a contradiction and completes the proof of the theorem. 


\section{The image of the Gauss map.}

Proposition. Let $M_{0}$ be a periodic minimal surface in $R^{3}$ having a fundamental domain of finite total curvature (nonzero) and let $M$ be a deformation of $M_{0}$, periodic for the same group. Then the Gauss maps of $M$ and $M_{0}$ have the same image, the complement of at most 4 points.

Proof. Considering the quotient spaces and §III, we know $\operatorname{deg} \bar{g}_{0}=\operatorname{deg} \bar{g}$ and $g$, $g_{0}$ have the same limiting values at the punctures. Therefore $g_{0}\left(M_{0}\right)=g(M)$. Now the proof follows Osserman's argument [4]; cf. [1]. We rotate $\tilde{M}$ (and conjugate $G$ ) so that $g$ takes finite nonzero values at the punctures and each pole of $g$ is simple. Let $q_{1}, \ldots, q_{k} \in S$ be the points missed by $g$, so $g^{-1}\left\{q_{1}, \ldots, q_{k}\right\} \subset\left\{p_{1}, \ldots, p_{r}\right\}$. Let $N=\operatorname{deg} g$ and at $p_{j}$ let $g$ assume its value with multiplicity $1+a_{j}$. Then

$$
K \cdot N \leqslant \sum_{j=1}^{r}\left(1+a_{j}\right)=r+\sum_{j=1}^{r} a_{j} .
$$

Let $\Omega=g^{\prime}(z) d z$ and $n=$ the total order of branching of $g=\#$ zeros of $\Omega$. At a simple pole of $g, \Omega$ has a double pole so \# poles of $\Omega=2 N$. By Riemann's relation:

$$
2 N-n=2-2 S \text {, }
$$

$S=$ the genus of $\bar{M}$. Now $\sum_{j=1}^{r} a_{j}$ is the order of branching at $p_{1}, \ldots, p_{r}$, hence $\leqslant n$. Thus

$$
K \cdot N \leqslant r+\sum_{j=1}^{r} a_{j} \leqslant r+n,
$$

and since $n=2(N+S-1)$ we have

$$
K \cdot N-r \leqslant 2(N+S-1) \text {. }
$$

Now $\omega$ has a pole at each puncture $p_{j}$ of order $v_{j}$ (by completeness), so by Riemann's relation:

$$
\sum_{j=1}^{r} v_{j}-\# \text { zeros of } \omega=2-2 S
$$

so

$$
r \leqslant \sum_{j=1}^{r} v_{j}=2-2 S+2 N .
$$

Combining (I) and (II) we have $K \leqslant 4$.

VI. The total curvature $4 \pi$ minimal surfaces in $R^{3} / Z$. Consider $N=R^{3} / Z$ as $R^{3}$ modulo one vertical translation and let $M \subset N$ be a c.m.s. of $C(M)=$ the total curvature of $M=4 \pi$. The helicoid is an example and the projection of the catenoid and Enneper's surface are others. We will now describe how such $M$ are obtained in general and exhibit new examples of periodic minimal surfaces in $R^{3}$, invariant under a vertical translation.

Let $(g, \omega)$ be a Weierstrass pair associated to $M$. Since $g: \bar{M} \rightarrow S$ is bijective we know $M$ is conformally a $K$-punctured sphere. We observe that $K \leqslant 4$. First perform a preliminary rotation of $M$ (and conjugate $G$ ) so that $g$ takes finite nonzero values at the punctures. Then $g$ has one simple pole in $M$ and no others in 
$\bar{M}$. Riemann's relation for $\omega$ is: \#poles of $\omega-\#$ zeros of $\omega=2$. Completeness of $M$ implies $\omega$ has poles at the punctures, and we know \#zeros of $\omega=2$, hence \# poles of $\omega=4$ and $K \leqslant 4$. Therefore $M$ had at most 4 punctures before we rotated. Now we consider each case $K=1,2,3,4$.

When $K=1, M$ is parametrised by a conformal map $X: \mathbf{C} \rightarrow M$ with harmonic coordinate functions and $X$ lifts to a conformal map $\tilde{X}: \mathbf{C} \rightarrow R^{3}$ which parametrises a c.m.s. $\tilde{M}$. The Gauss map of $\tilde{M}$ is also injective so $C(\tilde{M})=4 \pi$ and $\tilde{M}$ is a catenoid or Enneper's surface. Thus $M$ is the projection of one of these surfaces.

Consider now $K=2$. We parametrise $M$ by $\mathbf{C}^{*}=\mathbf{C}-0$ so 0 and $\infty$ correspond to the punctures. Suppose first that $g$ has a pole at $\infty$. We can reparametrise $M$ by composing $X$ with a Mobius transformation leaving 0 and $\infty$ fixed so that $g(z)=z+a$ for some $a \in \mathbf{C}$. Now $\omega$ is a meromorphic differential on $S$, analytic on $\mathbf{C}^{*}$ and having a pole at 0 , and no zeros in $\mathbf{C}$. Therefore $\omega=c d z / z^{n}$ for some integer $n \geqslant 1$ and $c \in \mathbf{C}^{*}$. The metric on $M$ is

$$
d s=\frac{|c|}{2|z|^{n}}\left(1+\left|z^{2}+2 a z+a^{2}\right|\right) .
$$

By completeness of $M$ we know that $\int_{\gamma} d s=\infty$ for every path $\gamma$ tending to $\infty$. This clearly implies $n<4$.

Let us first look at $n=1$, so $g(z)=z+a, \omega=c d z / z$. We have

$$
\begin{gathered}
\phi_{1}=\frac{c}{2 z}\left(1-z^{2}-2 a z-a^{2}\right), \quad \phi_{2}=\frac{i c}{2 z}\left(1+z^{2}+2 a z+a^{2}\right), \\
\phi_{3}=c(z+a) d z / z .
\end{gathered}
$$

The coordinate functions $x_{1}=\operatorname{Re} \int \phi_{1}$ and $x_{2}=\operatorname{Re} \int \phi_{2}$ are single valued on $M$, hence the residues of $\phi_{1}$ and $\phi_{2}$ at 0 are real. This implies $c\left(1-a^{2}\right)$ is real and $c\left(1+a^{2}\right)$ is pure imaginary. It is natural to ask that

$$
x_{3}=\operatorname{Re} \int \phi_{3}=\operatorname{Re}\left(c+c a \int d z / z\right)
$$

be multivalued on $M$ (otherwise the surface exists in $R^{3}$ ) hence the residue of $\phi_{3}$ at 0 should have a nonzero imaginary part; i.e. $\operatorname{Re}(2 \pi a c i) \neq 0$. Hence the conditions $c\left(1-a^{2}\right)$ real, $c\left(1+a^{2}\right)$ imaginary and $\operatorname{Re}(a c i) \neq 0$ determine all minimal surfaces in $R^{3} / Z$ of the type considered. For example, take $c=1, a=i$. We obtain

$$
\begin{aligned}
& x_{1}=\operatorname{Re}\left(\log z-\frac{z^{2}}{4}-i z\right), \\
& x_{2}=\operatorname{Re}\left(\frac{i z^{2}}{4}-z\right), \\
& x_{3}=\operatorname{Re}(z+i \log z) .
\end{aligned}
$$

To obtain the lifting $M$ of $\tilde{M}$ to $R^{3}$ we use the conformal covering map $e^{z}$ : $\mathbf{C} \rightarrow \mathbf{C}-0$ and

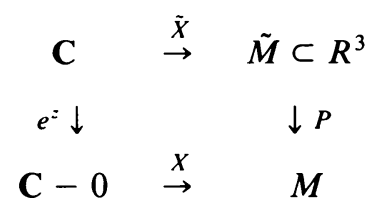


so the coordinate functions $\tilde{x}_{k}$ of $\tilde{X}$ are given by $\tilde{x}_{k}(z)=x_{k}\left(e^{z}\right)$ and $\tilde{g}=g\left(e^{z}\right)$, $\tilde{\omega}=\left(e^{z}\right)^{*} \omega$. Hence $\tilde{M}$ is the surface parametrised by

$$
\begin{aligned}
& \tilde{x}_{1}(z)=\operatorname{Re}\left(z-\frac{e^{2 z}}{4}-i e^{z}\right), \\
& \tilde{x}_{2}(z)=\operatorname{Re}\left(\frac{i e^{2 z}}{4}-e^{z}\right), \\
& \tilde{x}_{3}(z)=\operatorname{Re}\left(e^{z}+i z\right) .
\end{aligned}
$$

Writing $z=u+i v$, we obtain

$$
\begin{aligned}
& \tilde{x}_{1}=u-\frac{e^{2 u} \cos 2 v}{4}+e^{u} \sin v, \\
& \tilde{x}_{2}=-\frac{e^{2 u} \sin 2 v}{4}-e^{u} \sin v, \\
& \tilde{x}_{3}=e^{u} \cos v-v .
\end{aligned}
$$

Notice that replacing $v$ by $v+2 \pi$ and keeping $u$ fixed, changes $\tilde{x}_{3}$ by $-2 \pi$ and leaves $\tilde{x}_{1}, \tilde{x}_{2}$ fixed. Thus $\tilde{M}$ is a periodic surface, invariant by the vertical translation by $2 \pi$.

Next consider $n=2$, so $g(z)=z+a, \omega=c d z / z^{2}$. Letting $\Gamma$ denote the unit circle in $\mathbf{C}$ we have

$$
\int_{\Gamma} \phi_{1}=-a c 2 \pi i, \quad \int_{\Gamma} \phi_{2}=-a c \pi
$$

Since $x_{1}, x_{2}$ are single valued on $M$ we have $a c$ is real and pure imaginary, thus $a=0$. Notice that $\int_{\Gamma} \phi_{3}=2 \pi i c$, so if this surface is to exist genuinely in $R^{3} / Z$ we must have $\operatorname{Im}(c) \neq 0$. Now a calculation yields:

$$
\begin{aligned}
& \tilde{x}_{1}=-r \cos v \cosh u+s \sin v \sinh u, \\
& \tilde{x}_{2}=-s \cos v \sinh u-r \sin v \cosh u, \\
& \tilde{x}_{3}=r u-s v,
\end{aligned}
$$

where $c=r+i s, z=u+i v$. For $r=0$ this is a helicoid and for $s=0$ a catenoid. For $c=e^{i \theta}, 0 \leqslant \theta \leqslant \pi / 2$, we obtain the usual deformation of the helicoid into its associate surfaces. Whenever $s \neq 0$, the surface is invariant by vertical translation by $2 \pi$; changing $v$ by $2 \pi$ does not change $\tilde{x}_{1}, \tilde{x}_{2}$.

The case $n=3$ is impossible since then $\int_{\Gamma} \phi_{1}=-2 \pi i c$ and $\int_{\Gamma} \phi_{2}=-2 \pi c$; therefore $c$ would be both real and imaginary, hence 0 .

To complete the study of two punctures it remains to consider the case when $g$ takes a finite value at $\infty$. We reparametrise $M$ by $\mathbf{C}-0$ so that

$$
g(z)=\frac{a z+b}{z+d}
$$

so that $g$ has a simple pole at $-d$. We know $\omega=(P(z) / Q(z)) d z$, where $P$ has a double zero at $-d$ if $d \neq 0$ and $Q$ can vanish only at 0 . Suppose first that $d=0$ so $\omega=c z^{m} d z$ for some integer $m$. If $m \geqslant 2$, then $\phi_{k}, k=1,2,3$, are analytic at 0 , so $M$ is parametrised by $\mathbf{C}$ and we know by the previous case what $M$ is, the projection 
of a catenoid or Enneper's surface. Suppose $m=0$. Then calculating residues of $\phi_{1}$ and $\phi_{2}$ at 0 and using the fact that $x_{1}, x_{2}$ are single valued we conclude $a b c$ is real and imaginary, hence $a=0$ or $b=0$. Clearly $b \neq 0$ since $g$ is injective, hence $a=0$. The residue of $\phi_{3}$ at 0 is $c b$ so $\operatorname{Im}(c b)=0$ if $x_{3}$ is to be multivalued; otherwise $M$ is the projection of a catenoid since $\operatorname{Res}\left(\phi_{1}\right)=\operatorname{Res}\left(\phi_{2}\right)=0$, so $M$ lifts to $R^{3}$, parametrised by $\mathrm{C}-0$ and of total curvature $4 \pi$. A calculation yields

$$
\begin{aligned}
& \tilde{x}_{1}=\operatorname{Re}\left(\frac{c}{2}\left(e^{z}+b^{2} e^{-z}\right)\right), \\
& \tilde{x}_{2}=\operatorname{Re}\left(\frac{i c}{2}\left(e^{z}-b^{2} e^{-z}\right)\right), \\
& \tilde{x}_{3}=\operatorname{Re}(c b z) .
\end{aligned}
$$

These are the "associate type" surfaces of the helicoid (the helicoid is $c=i, b=1$ ). Now suppose $g(z)=b / z, \omega=c z d z$. Then $\operatorname{Res} \phi_{1}=-c b^{2} / 2$ and $\operatorname{Res}\left(\phi_{2}\right)=i c b^{2}$, so $c b^{2}$ is real and imaginary, hence $b=0$ which is impossible.

Next we study $d \neq 0$. Since $g$ is analytic at $0, \omega$ must have a pole at 0 by completeness of $M$. Also $\omega$ has a double zero at $-d$ since $g$ has a simple pole there. So $\omega=c(z+d)^{2} d z / z^{n}$ for some $c \in \mathbf{C}-0$ and $n \geqslant 1$. We have

$$
d s=\left.\left|\frac{c(z+d)^{2}}{2 z^{n}}\right||1+| \frac{a z+b}{z+d}\right|^{2}|| d z \mid .
$$

Completeness at $\infty$ implies $n<4$. If $n=1$, then calculating residues of $\phi_{1}, \phi_{2}$ at 0 yield $c\left(d^{2}-b^{2}\right)$ is real and $c\left(d^{2}+b^{2}\right)$ is imaginary (using the fact that $x_{1}, x_{2}$ are single valued). We want $x_{3}$ to be multivalued which yields $\operatorname{Im}(c b d) \neq 0$. Such surfaces are easily realised, e.g., $d^{2}=\bar{b}^{2}$ and $c$ imaginary. When $n=2$ the analogous calculations yield $c(d-a b)$ real, $c(d+a b)$ imaginary and $\operatorname{Im}(c(b+d a)) \neq 0$. For $n=3, c\left(1-a^{2}\right)$ real, $c\left(1+a^{2}\right)$ imaginary and $\operatorname{Im}(a c) \neq 0$; e.g., $a=1, c$ imaginary. This completes the study of two punctures.

We will not treat the 3- and 4-puncture case in detail; we will make some general remarks and give some examples. Consider the 3-puncture case. Since there is only one conformal structure on a 3-punctured sphere, we may parametrise $M$.by $S-\left\{\theta_{1}, \theta_{2}, \theta_{3}\right\}$, where $\theta_{k}$ are the cube roots of unity. Now $g(z)=(a z+b) /(z+d)$ and we suppose $d \neq-\theta_{k}, k=1,2,3$. Then $\omega$ has a double zero at $-d$ and a pole at each $\theta_{k}$, so

$$
\omega=\left(c(z+d)^{2}\right) /\left(\left(z-\theta_{1}\right)^{n_{1}}\left(z-\theta_{2}\right)^{n_{2}}\left(z-\theta_{3}\right)^{n_{3}}\right) .
$$

Since $\infty$ is a regular point of $M$ and $\omega$ is not zero there we have $n_{1}+n_{2}+n_{3} \leqslant 4$, so there are several cases to consider (we will not do it). In order that $M$ exist, one must choose $a, b, c, d$ so that the residues of $\phi_{1}, \phi_{2}$ are real at each puncture and the imaginary parts of the residues of $\phi_{3}$ at each puncture must generate a nontrivial cyclic group. If we assume $\infty$ is a pole of $g$, the situation is simpler. Then $g(z)=a z+b$ and

$$
\omega=(c d z) /\left(\left(z-\theta_{1}\right)^{n_{1}}\left(z-\theta_{2}\right)^{n_{2}}\left(z-\theta_{3}\right)^{n_{3}}\right) .
$$

Now $\infty$ is a regular point of $M$, so letting $u=1 / z, \omega(u)$ must have a double pole at 
0 . This yields $n_{1}+n_{2}+n_{3}=4$. One can analyse this situation completely. For example taking $a=1, b=0, c$ real and $n_{1}=2, n_{2}=n_{3}=1$, a (tedious) calculation shows this surface exists in $R^{3} / Z$.

When $M$ is a 4-punctured sphere, the conformal structure may change. One can check the following example exists:

$$
\begin{gathered}
g(z)=z, \quad c=4 i, \quad \text { and } \omega=\frac{c d z}{(z-1)(z-i)(z+1)(z+i)}, \\
M \text { is } S-\{1,-1, i,-i\} .
\end{gathered}
$$

Can every conformal structure be realised? Let $z_{0} \in \mathbf{C}$ and $g(z)=a z+b, \omega=$ $c d z /\left(z-z_{0}\right)\left(z_{0}+1\right)\left(z_{0}-i\right)\left(z_{0}+i\right)$. Can one choose $a, b$ and $c$ so that $M=S-$ $\left\{z_{0},-1, i,-i\right\}$ exists?

\section{Some isolated periodic surfaces.}

Proposition. Let $\tilde{M}_{0}$ be the usual helicoid in $R^{3}: \tilde{g}_{0}(z)=+i e^{z}, \tilde{\omega}_{0}=e^{-z} d z$. Then $\tilde{M}_{0}$ is isolated among complete minimal surfaces invariant under vertical translation by $2 \pi$.

Proof. Let $M_{0}$ be the projection of $\tilde{M}_{0}$ to $R^{3} / Z$, so that $C\left(M_{0}\right)=4 \pi, M_{0}$ is a 2-punctured sphere and $g_{0}(z)=i z, \omega_{0}=d z / z^{2}$. A deformation $M$ of $\tilde{M}_{0}$ invariant under vertical translation by $2 \pi$ gives a deformation $M$ of $M_{0}$ in $R^{3} / Z$. $M$ is also conformally a 2-punctured sphere and the Gauss map $g$ of $M$ is bijective. We parametrise $M$ by $\mathbf{C}-0$. The limiting values of $g$ and $g_{0}$ are the same at the punctures 0 and $\infty$ so we can parametrise $M$ so that $g(z)=i z$. Now $\omega$ is a meromorphic differential on $S$ with no zeros in $\mathbf{C}-0$ and a pole at 0 , hence $\omega=c d z / z^{k}$ for some $c \in \mathbf{C}, k \geqslant 1$. The metric on $M$ is

$$
d s=\frac{|c|}{\left|z^{k}\right|}\left(1+|z|^{2}\right)
$$

so by completeness of $M$ at $\infty$ we have $k \leqslant 3$. If $k=1$, then $\phi_{1}=c\left(1+z^{2}\right) / 2 z$ and $\phi_{2}=i c\left(1-z^{2}\right) / 2 z$. Hence $\operatorname{Res}\left(\phi_{1}, 0\right)=c / 2$ and $\operatorname{Res}\left(\phi_{2}, 0\right)=i c / 2$. Since $x_{1}=$ $\operatorname{Re} \int \phi_{1}, x_{2}=\operatorname{Re} \int \phi_{2}$ are single valued functions on $M, c$ is both real and imaginary, so $c=0$, a contradiction. A similar reasoning shows $k=3$ is impossible, and $\omega=c d z / z^{2}$.

We have $\operatorname{Res}\left(\phi_{3}, 0\right)=-c 2 \pi$ so $\operatorname{Re}(c)= \pm 1$. Now lift $M$ to $\tilde{M} \subset R^{3}$ by the conformal covering $e^{z}: \mathbf{C} \rightarrow \mathbf{C}-0$. This yields $\tilde{g}=g\left(e^{z}\right), \tilde{\omega}=\left(e^{z}\right)^{*} \omega$ and a calculation shows (writing $c=a+i b$ )

$$
\begin{aligned}
& \tilde{x}_{1}=a \cos v \sinh u-b \sin v \cosh u, \\
& \tilde{x}_{2}=b \cos v \cosh u+a \sin v \sinh u, \\
& \tilde{x}_{3}=-(b u+a v) .
\end{aligned}
$$

Considering the trace of $\tilde{M}$ in the plane $\tilde{x}_{3}=0$ it is clear that $b=0$ since $\tilde{M}$ is a deformation of $\tilde{M}_{0} \cdot{ }^{1}$ Thus $\tilde{M}$ is also a helicoid.

\footnotetext{
${ }^{1}$ The surface is a homothety of an associate surface of the helicoid and is not embedded for $b \neq 0$.
} 
Proposition. The surface $M_{0}: g_{0}(z)=z, \omega_{0}=4 i d z /\left(z^{4}-1\right)$ (modelled on $S$ punctured at the 4 th roots of unity) is isolated in $R^{3} / Z$.

Proof. Let $M$ be a deformation of $M_{0}$. The total curvature of $M$ is $4 \pi$ so $\bar{g}$ is bijective. Since $\bar{g}$ at the punctures misses precisely the 4th roots of unity $1,-1, i,-i$, $g$ is a conformal equivalence between $M$ and $S-\{1,-1, i,-i\}=S_{0}$. Hence we may parametrise $M$ by $S_{0}$ so that $g(z)=z$. Then $\omega$ is a meromorphic differential on $S$ with no zeros in $\mathbf{C}$ and poles at $\{1,-1, i,-i\}$, so

$$
\omega=\frac{c d z}{(z-1)^{n_{1}}(z+1)^{n_{2}}(z-i)^{n_{3}}(z+i)^{n_{4}}} .
$$

Since $\infty$ is a simple pole of $g$, it is a double zero of $\omega$, hence $n_{1}+n_{2}+n_{3}+n_{4}=4$. Thus $\omega=c d z /\left(z^{4}-1\right)$. We have the residue at $\phi_{3}$ at each puncture is $\pm c / 4$, and since $M$ is invariant by vertical translation by $2 \pi$, the real part of $\pi i c / 2= \pm 2 \pi$, i.e. $\operatorname{Im}(c)= \pm 4$. Now $\operatorname{Res}\left(\phi_{1}, i\right)=i c / 2$ and $x_{1}$ is single valued on $M$ so $c$ is pure imaginary. Hence $M=M_{0}$.

Similar arguments apply to other $4 \pi$ total curvature minimal surfaces in translation spaces; e.g. Scherk's surface, $g(z)=z, \omega=2 d z /\left(z^{4}-1\right)$, is isolated in $R^{3} / G$, $G$ the group generated by translation by $(2 \pi, 0,0)$ and $(0,2 \pi, 0)$.

We have a partial result concerning deformations of the helicoid which are close at $\infty$.

Proposition. Let $M$ be a deformation of the helicoid $M_{0}$ satisfying:

1. $M$ is parabolic,

2. $M$ is transverse to every horizontal plane $x_{3}=$ constant,

3. the curve $M \cap\left\{x_{3}=\right.$ constant $\}$ makes a bounded angle with $M_{0} \cap\left\{x_{3}=\right.$ constant $\}$, the bound independent of the plane $x_{3}=$ constant .

Then $M$ is a helicoid.

Proof. Let $\sigma: M \rightarrow R$ be the angle which the curve $M \cap\left\{x_{3}=\right.$ constant $\}$ makes with the vector $(1,0,0)$. A priori, $\sigma$ is a local function on $M$ but since $M$ is simply connected and $M$ is transverse to every horizontal plane, we can extend $\sigma$ to a continuous (unbounded) function on $M$. It is a general fact that $\sigma$ is a harmonic function on $M$ [5]. Now $M$ is a minimal surface so $x_{3}$ is also a harmonic function on $M$. Therefore $x_{3}-\sigma$ is a harmonic function on $M$ which is bounded by condition 2 . But by condition $1, M$ is conformally parametrised by $\mathbf{C}$, so $x_{3}-\sigma$ is constant. Hence for $x_{3}=$ constant we have $\sigma=$ constant, i.e., $M \cap\left\{x_{3}=\right.$ constant $\}$ is a straight line. Therefore $M$ is a helicoid.

REMARKS. 1. If $\varepsilon$ is small enough, then $M$ is certainly parabolic since the orthogonal projection is a quasiconformal homeomorphism and $M_{0}$ is conformally parametrised by $\mathbf{C}$.

2. It is elementary that for any minimal surface $M$ there is a positive function $\varepsilon(x)$ on $M$ such that any deformation of $M$ which is $\varepsilon(x)$-close to $M$, is equal to $M$. 


\section{BIBLIOGRAPHY}

1. F. Gacksatter, Über Abelshe Minimalflächen, Math. Nachr. 74 (1976), 157-165.

2. B. Lawson, Lectures on minimal submanifolds, vol. 1, Math. Lecture Series 9, Publish or Perish, Berkeley, Calif., 1980.

3. W. Meeks, A survey of the geometric results in the classical theory of minimal surfaces, Bol. Soc. Brasil. Mat. 12 (1981), 29-86.

4. R. Osserman, A survey of minimal surfaces, Van Nostrand-Reinhold, New York, 1969.

5. M. Shiffman, On surfaces of stationary area bounded by two circles, or convex curves, in parallel planes, Ann. of Math. (2) 63 (1956), 77-90.

6. F. Xavier, The Gauss map of a complete non flat minimal surface cannot omit 7 points of the sphere, Ann. of Math. 113 (1981), 211-214.

Departement de Mathematiques, Universite de Paris ViI, 2 Place Jussieu, 75005 Paris, France 